\title{
Stabilization Methods in the Submerged Arc Discharge Synthesis of Carbon Nanostructures
}

\author{
Lorenzo Hernandez-Tabares $\mathbb{D}^{1},{ }^{1}$ Juan G. Darias-Gonzalez $\mathbb{D}^{1},{ }^{1}$ Frank J. Chao-Mujica $\mathbb{D}^{1}{ }^{1}$ \\ Luis M. Ledo-Pereda $\left(\mathbb{D},{ }^{1}\right.$ Manuel Antuch $\left(\mathbb{D},{ }^{1}\right.$ Ernesto Carrillo-Barroso $(\mathbb{D}){ }^{2}$ \\ J. Enrique Chong-Quero $\mathbb{D}^{\mathbb{D}},{ }^{3}$ Edilso Reguera $\mathbb{D}^{4},{ }^{4}$ and Luis F. Desdin-Garcia $\mathbb{D}^{1}$
}

${ }^{1}$ Grupo de Nanociencia, Centro de Aplicaciones Tecnológicas y Desarrollo Nuclear (CEADEN), La Habana 11300, Cuba

${ }^{2}$ Departamento de Física Aplicada, Instituto de Cibernética, Matemática y Física, La Habana 10400, Cuba

${ }^{3}$ Escuela de Ingeniería y Ciencias, Tecnologico de Monterrey, Edo. de Mex, 52926, Mexico

${ }^{4}$ Laboratorio Nacional de Almacenamiento y Conversión de Energía, Instituto Politécnico Nacional, Ciudad de México 11500, Mexico

Correspondence should be addressed to J. Enrique Chong-Quero; jchong@tec.mx, Edilso Reguera; edilso.reguera@gmail.com, and Luis F. Desdin-Garcia; desdin@ceaden.edu.cu

Received 3 June 2021; Accepted 19 October 2021; Published 8 November 2021

Academic Editor: Filippo Giubileo

Copyright (c) 2021 Lorenzo Hernandez-Tabares et al. This is an open access article distributed under the Creative Commons Attribution License, which permits unrestricted use, distribution, and reproduction in any medium, provided the original work is properly cited.

\begin{abstract}
Many papers, in which the submerged arc discharge (SAD) method in nanoparticle synthesis was used, reported similar operating parameters, but different electrode erosion rate values, different yields and purities of the obtained nanostructures, and a different sort of contaminants present in the synthesis. Analyzing these articles, we found insufficient attention to ensure the arc power stability, which is a key factor guaranteeing the product homogeneity and quality. This paper presents an analysis of different control strategies, remarks their advantages and drawbacks, and proposes the most appropriate technique to be used in SAD. The most appropriate technique is proposed from the SAD stabilization method analysis.
\end{abstract}

\section{Introduction}

Carbon nanostructures (CNSs) have a remarkable use in nanoscience because of their exceptional thermal, electrical, chemical, and mechanical properties. CNSs that have been manufactured keeping under control the structure in nanometer scale and the binding nature of carbon atoms can lead to highly functional advanced performances, which are hard to achieve using conventional carbons $[1,2]$. These CNSs have found application in areas as diverse as composite materials, energy storage and conversion, sensors, drug delivery, catalyst support, paints, nanoelectronics, and gas storage, among others [3]. However, one of the issues that still need to be improved in CNS synthesis is the necessity to obtain high yields through simple synthesis processes.
Among the methods used to produce CNSs like fullerenes and nanotubes, those based on carbon sublimation at elevated temperatures (laser ablation and arc discharge), followed by reaggregation in the solid state at cooler regions, play a significant role.

Intending to scale up the continuous production of CNSs, the arc-discharge method in gases was adapted to liquid environments, consequently avoiding the utilization of vacuum systems and inert atmospheres. Submerged arc discharge (SAD) is one of the most employed techniques for synthesizing several types of CNSs [4-10]. Figure 1 shows the main carbon nanostructures and contaminants produced by SAD, namely, multiwall carbon nanotubes (MWCNT), single-wall carbon nanotubes (SWCNT), carbon nanoonions $(\mathrm{CNO})$, carbon nanohorns $(\mathrm{CNH})$, carbon 


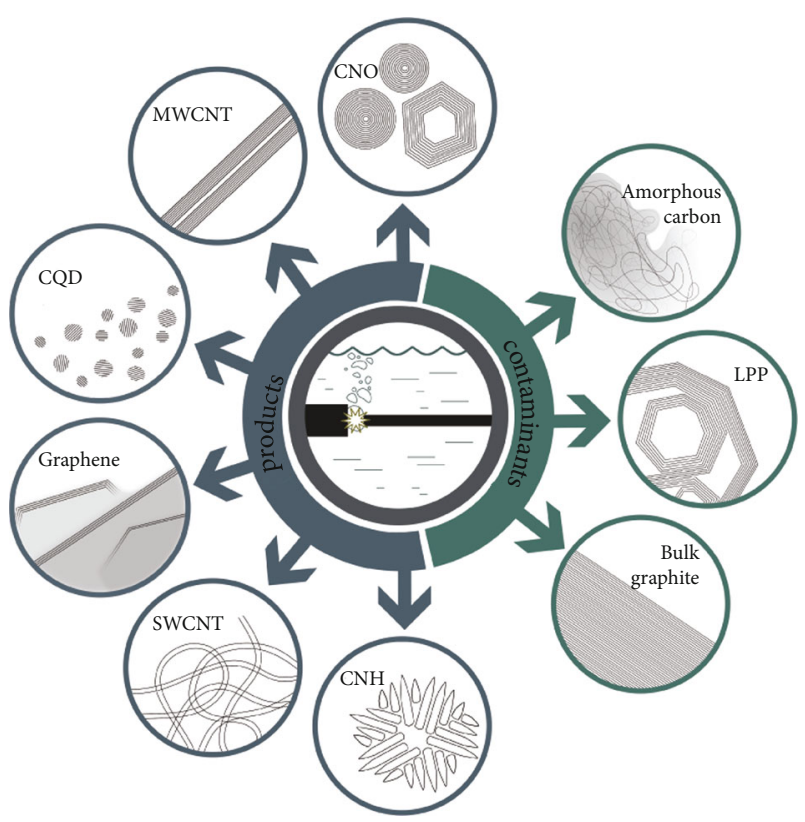

FIGURE 1: Main carbon nanostructures and contaminants produced by SAD.

quantum dots (CQD), oxidized graphene sheets, large polyhedral particles (LPP), amorphous carbon, and graphite particles.

Although this technique is straightforward and uses standard equipment, several factors make it difficult to control the arc power stability, which is something that is more difficult to achieve in liquid than in gas [11]. This stability is essential to guarantee the product homogeneity and quality. The gas bubbles created within the liquid act as reactor chambers [12] allowing the fast quenching of carbon vapors; hence, it is the bubble's volume that defines the size of the synthesized nanoparticles. However, this volume depends on the electric arc power $[13,14]$. Consequently, a fluctuating arc power increases the nanoparticle size dispersion and modifies their structures. However, while many papers have not considered this, others have reported the use of different stabilization strategies (Figure 2).

Analyzing the most cited papers, we identified three main groups: (a) without stabilization, (b) manual stabilization, and (c) automatic stabilization (Figure 3 ).

In the first group, the arc is ignited by putting the electrodes in physical contact. Subsequently, the electrodes are separated again and kept in a fixed position until the arc is turned off as a result of the electrode wear. However, because there is no rectification of the electrode gap or the arc current, the arc power is not constant and gradually changes as the electrode is being eroded. Furthermore, the synthesis times are less than 2 min [15-19].

In the second group, an operator tries to maintain a constant electrode gap or a constant arc current value by employing manual control $[13,20,21]$. In Figure $3,77 \%$ of the works did not employ any automatic stabilization procedure.

In the last group, process control through a feedback signal is applied. A characteristic physical magnitude of the process (e.g., arc current, arc voltage, and emitted radiation)

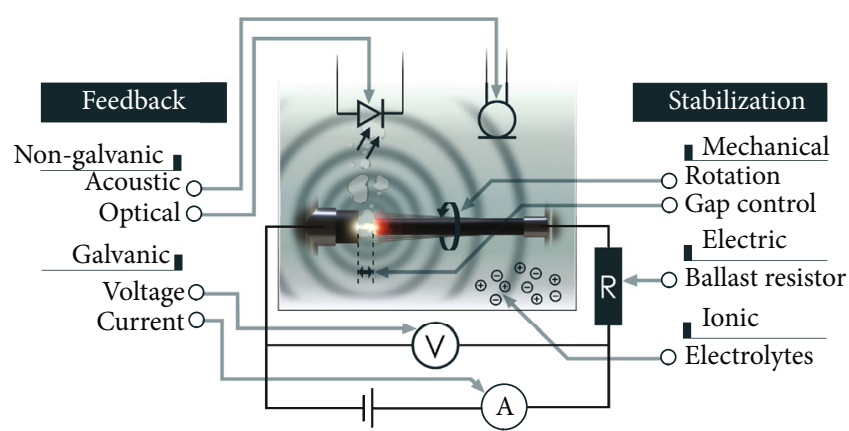

FIGURE 2: Scheme of the submerged arc discharge circuit, control strategies, and feedback signals.

is used as a feedback signal. While the emitted radiation allows the implementation of a nongalvanic feedback, electrical parameters are used to implement a galvanic one. The synergy of these techniques is employed in some cases.

Aside from the three main groups, other arc stabilization strategies (e.g., electrode rotation) for achieving a more uniform erosion or inclusion in the circuit of a ballast resistor have also been reported. In [22], a better arc stabilization was reported as a consequence of modifying the conductivity of the liquid environment by adding salts.

Other works have claimed a better route for control and stabilization using pulsed and AC arc techniques [23-25]. The present work focuses only on submerged DC arc discharge.

Articles usually do not mention what specific type of power supply was employed, reporting in many cases the use of arc welding machines, which adds difficulty in experiment replication.

The above discussion illustrates the complexity of the arc discharge in liquids, the difficulties involved in achieving its stability, and the importance of the latter in the quality of the obtained CNSs.

Therefore, the insufficient attention given to ensure the arc power stability in SAD and the different approaches to do so could explain why existing articles, although reporting similar operating parameters, show the following differences: (a) the electrode erosion rate values are scarce and contradictory, (b) no rigorous yield value has yet been reported, and (c) the nanostructure purity degree and the contaminants sorts present in the synthesis radically vary. As far as we know, a comparative analysis of the different approaches in stabilization strategies has not yet been performed.

The abovementioned shortages point out the pertinence of performing an analysis of the stabilization methods used in synthesizing CNSs by SAD. In this study, such an analysis is conducted based on the experiments performed by the authors and by examining other pertinent papers involving optoelectronic, acoustic, voltage, current, and ballast resistance methods. An analysis of different control strategies is presented herein, showing their advantages and drawbacks. The most appropriate method to be used in SAD is then proposed. This study will focus only on SAD using direct current. The set-up mechanical design influence will not be considered. 


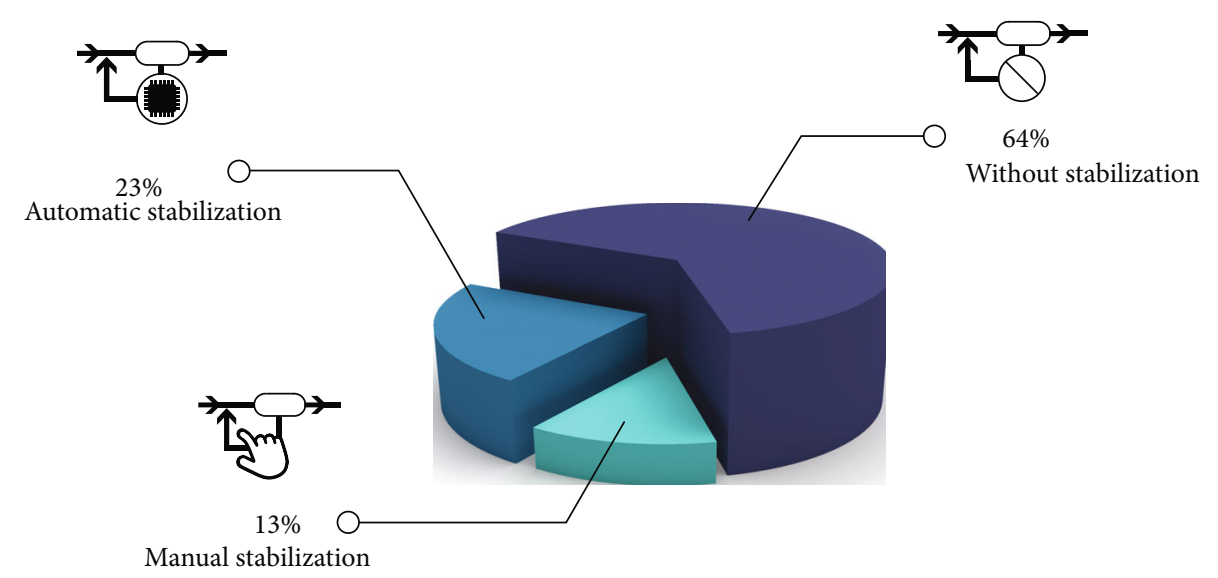

FIgure 3: Three main groups of the SAD experiments.

The first section presents published articles that report similar operating parameters, but contradictory results. The second section describes the main SAD instability sources. The third section explains the fundamentals of the reported stabilization methods in the SAD. The fourth section discusses the advantages and disadvantages of the SAD stabilization methods. Lastly, the last section draws the conclusions.

\section{Instability Sources in the Submerged Arc Discharge}

In an electric arc discharge, several factors disturb the arc current and the power stability. While some of them are common for both gaseous and submerged discharges, the liquid environment provides additional difficulties for others (Figure 4).

The key differences between SAD and conventional arc discharge in gases are the gaseous components surrounding the arc spot and the quenching rate of the evaporated carbon vapor [13]. In SAD, once the electric arc starts, a gaseous environment in the form of a bubble is created around the arc. The typically reported pressure inside the bubble goes from $0.5 \mathrm{~atm}$. to $1 \mathrm{~atm}$; hence, the arc is classified as a highpressure arc. Among arcs of this sort, including those in open air, those at atmospheric pressure are especially frequent. The bubble's gas-liquid interface acts as a container wall. If the liquid is water, the bubbles are basically composed of carbon vapor, water vapor, hydrogen, and carbon monoxide [26].

2.1. Lower Cathode Temperatures and Strong Bubbling. The current density at the cathode is governed by Richardson's law [27]; thus, it has a strong dependence on the cathode temperature. In SAD, the cathode is surrounded by liquid; therefore, it experiences much more intense cooling than in gas discharges. In contrast, in the vicinity of the arc, the temperature gradient is very high, and a strong bubbling is caused by the gas generated in the chemical reactions and liquid evaporation. This bubbling produces an additional temperature fluctuation in the cathode. Furthermore, the rapid temperature variation influences the recombination of the ionic species present in the discharge channel leading to conductivity oscillation.
2.2. Electrode Heterogeneity. As a rule, the electrodes are assumed to have a negligible degree of heterogeneity. However, they always entail a degree of intrinsic heterogeneity because they are manufactured by sintering fine grains of graphite. Therefore, different values of flexural strength and thermal and electrical conductivities, among other parameters, can be observed in different electrode zones [28]. This factor is one of the causes that make the SAD technique unfree of contaminants [29].

2.3. Vibrations. Another important fact is that the anode and its holder undergo vibrations. These vibrations and the high temperatures on the anode surface cause the detachment of graphite particles [30], which increases the contaminant by-products. When entering the discharge channel, these particles cool the gas, increase the ion-electron recombination, and alter the channel resistivity [31]. Next, when evaporating, they increase the number of charge carriers and decrease the interelectrode potential. Consequently, the arc current oscillations are related with the vibrations of the anode and its holder.

2.4. Power Supply. As in any other electric circuit, the power supply should satisfy the demanded current at the given voltage. A power supply with insufficient filtering and stabilization technique makes the arc power fluctuate. Although many papers have used welding machines as power supplies, these machines are not power supplies. They work under different working principles. In many cases, they are unregulated sources. Sometimes, how their control systems work is unknown. These facts compromise the total control and repeatability of experiments; hence, the synthesis results can vary depending on the machine used. Furthermore, the power supply's output impedance should be considered when planning the appropriate working parameters. This will be discussed later.

\section{Submerged Arc Discharge Stabilization Methods}

As exposed before, different strategies have been used to stabilize the arc discharge or to achieve a sufficiently stable arc 


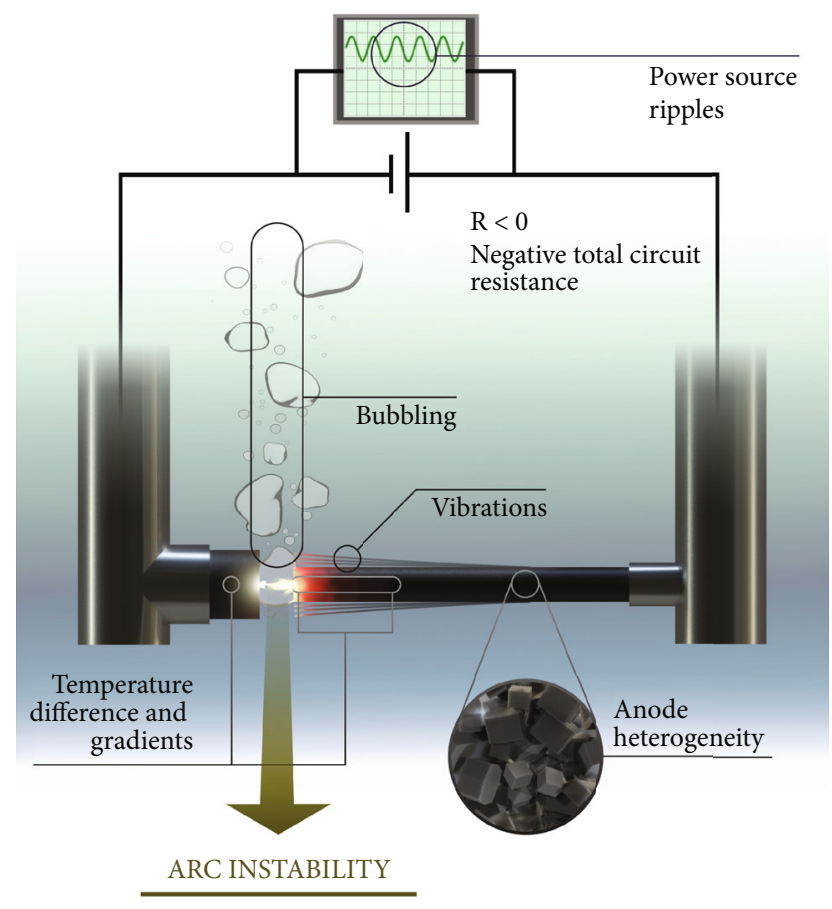

FIGURE 4: Main instability sources in the submerged arc discharge.

for performing experiments. This section will be devoted to explaining the fundamentals of the reported stabilization methods in SAD.

3.1. Electrolytes. Using electrolytes to improve stability through conductivity increment has been accompanied by other purposes. They have been used to obtain more ordered or decorated nanostructures and increase the liquid's cooling capacity. The synthesis of SWCNTs and MWCNTs through arc-discharge in the $\mathrm{H}_{3} \mathrm{VO}_{4}$ aqueous solution was reported in [20] based on the vanadium compound solution properties of stimulating the formation of more ordered CNSs. CNTs filled with metallic cobalt and elemental sulfur were obtained in the $\mathrm{CoSO}_{4}$ solution [26]. A simple and one-step SAD synthesis route for producing palladium-decorated MWCNTs was presented in [32]. Under different thermal conditions, CNTs were produced by SAD in the $\mathrm{LiCl}$ solution [33]. The impact of the solution temperature on the nanoproduct structures was also discussed. In [22], a $\mathrm{NaCl}$ solution was used to attain a better cooling ability of the liquid and increase its electrical conductivity.

3.2. Electrode Rotation. The plasma-rotating electrode process (PREP) has been used in different gas atmospheres (i.e., air, $\mathrm{N}_{2}, \mathrm{H}_{2}$, He, and Ar), pressures (i.e., 40-700 Torr), rotation speeds (i.e., 0-10,000 rpm), and current ranges (i.e., $40-200 \mathrm{~A})$ to synthesize SWCNH, SWCNTs, MWCNTs, and highly convoluted graphene sheets [34-43]. The authors claim two improvements: (1) the rotating anode spreads the microdischarges between the electrodes more uniformly, thereby stabilizing the plasma, more homogeneously eroding the anode, and increasing the continuous operation time; and (2) a centrifugal turbulence engendered by the fast electrode rotation induces the vaporized material to shift away and build up on the chamber's walls or collectors rather than be restricted to the cathode surface. The PREP in deionized water was reported in [4]. It was used for the selective synthesis of turbostratic polyhedral CNOs. The best-operating conditions were reported at $30 \mathrm{~A}, 12 \mathrm{~V}$, and $20 \mathrm{rpm}$ rotation speed. An approximately $3 \mathrm{~mm}$-diameter cylindrical black hard accretion that is weakly fixed to the cathode surface was created and removed by applying a weak lateral force. This material is enriched in CNOs and must be processed in a laborious way for its extraction.

3.3. Electrodes at Constant Speed. In some studies, electrodes were forced to approach each other at a constant speed to try to stabilize the discharge $[34,44]$. Based on automatic welding machines that move the electrode at a constant speed to obtain a uniform welding cord, and supposing that such movement could lead to a constant arc power or gap between electrodes, a constant speed control algorithm was tested in [45]. For better stability, this method was combined with electrode rotation [34].

3.4. SAD without any Control Technique. As mentioned before, the majority of the reported works on SAD did not use any stabilization technique. To evaluate the type of SAD, an experiment was performed using the installation described in [45]. The anode movement was, however, stopped once the discharge was initiated. As the anode wore out, the gap between the electrodes increased, and the arc power changed until the discharge was extinguished. The experiment was repeated for five times at $30 \mathrm{~A}$ arc current. Figure 5 presents the arc power behavior as a function of time of one of the runs. The moment, at which the anode movement was stopped, was used as the starting time. The extinction time is the interval from 0 to $\tau$ ( $\tau$ is the moment at which the transition from a hissing arc to a silent arc occurs). The silent arc regime is the time interval $\tau-\tau_{o}$. The CNSs were not synthesized in the silent arc. A mean arc extinction time $\tau$ of $6.35 \pm$ $1.72 \mathrm{~s}$ and an energy consumption $E$ of $3800 \pm 1096 \mathrm{~J}$, which was a relatively large dispersion $(\sim 30 \%)$, were calculated. In the 0 to $\tau$ time interval, the power oscillations ( $\left.P_{\max }-P_{\min }\right) / P_{\min }$ reached a maximum of $38 \%$, indicating a big instability. The $\tau$ value depends on the electrical parameters of the system and the used electrodes. For example, in [15], with an initial power of $1500 \mathrm{~W}$ and anode and cathode diameters of $7 \mathrm{~mm}$ and $20 \mathrm{~mm}$, respectively, the discharge was maintained for $97 \mathrm{~s}$.

3.5. SAD with Manual Control. The gap was manually controlled in a considerable number of published articles. The control was based on the appreciation of the emitted arc light intensity or the measuring device indications.

3.6. Automatic Control (Galvanic and Nongalvanic Feedback Stabilization Methods). When implementing a closed-loop automatic control system, a physical variable that characterizes the process must be monitored. The arc-emitted 


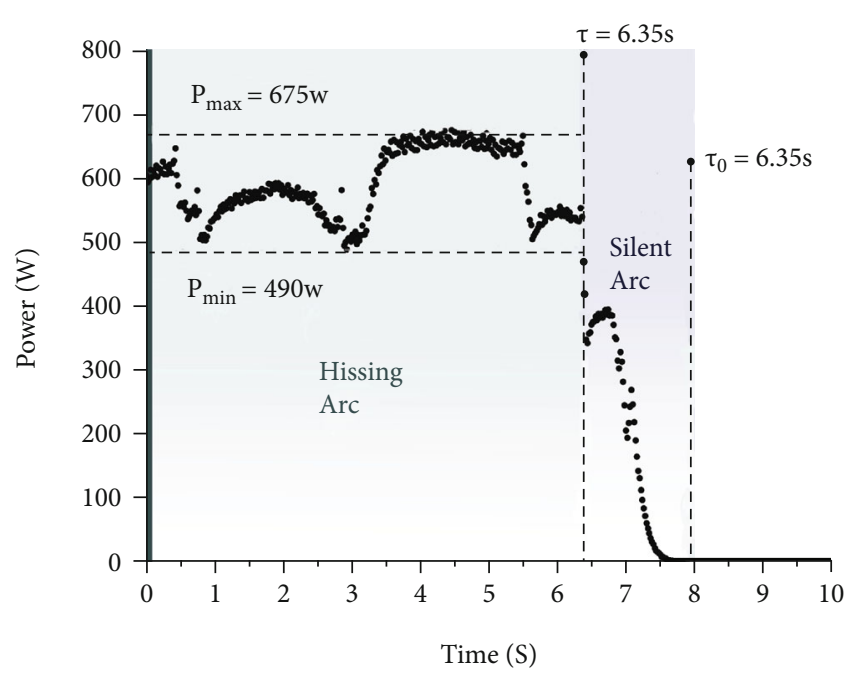

FIGURE 5: Extinction of the arc power as a function of time.

light and sound, arc current, and voltage are some of the variables sensed in experiments. Depending on the variable type, galvanic isolation may or may not exist between the control system and the process to be controlled. The optoelectronic and acoustic approaches are among the possible nongalvanic feedback stabilization methods. Both are well shielded against electromagnetic fields, and the detected signals are directly related to the arc. On the contrary, electrical parameters like arc current or voltage have been used to implement galvanic feedback control.

3.6.1. Optical Control. Arc discharge optical emission has been used to determine the carbon dimmers precursor concentrations and the different arc zone temperatures [31, 41, 43-48]. However, it has been almost exclusively employed in controlling the arc discharges in gases and mainly in fullerene synthesis [49]. A SAD stability control based on light emission has been scantily reported. Accordingly, [50, 51] described the temporal relationship in SAD among the arc light, voltage, and current. The arc light was also analyzed through optical emission spectroscopy. In addition, some papers reported the optoelectronic control of the arc discharge $[11,32,52]$.

The optoelectronic control should satisfy basic requirements. The arc light emission $\Phi$ must be isotropically radiated from the gap in a plane $Z$ perpendicular to the electrode axis $X$ (Figure 6(a)). Given the small gap size, using a plane not perpendicular to the $x$-axis can lead to the electrodes hindering light passage. Therefore, for the solid angle $\Omega$ (gap-detector surface), parameter $\alpha$ (degree of isotropy of the light emission by the arc) should be constant for each given value of $\theta(1)$, where $\theta$ is the rotation angle to the $x$-axis. The optical emission must be correlated with the arc discharge current ( $\Gamma=$ const, (2)). The transmittance $T$ during the synthesis must be constant (3). Moreover, the possibility of optically controlling the arc power through a certain dedicated spectrum line is desirable.

$$
\begin{gathered}
\alpha=\frac{\Phi(\theta)}{\int_{0}^{2 \pi} \Phi(\theta) d \theta}=\text { const. } \\
\Gamma=\frac{I(t)}{\int_{0}^{2 \pi} \Phi(\theta, t) d \theta}=\text { const. } \\
T=\text { const. }
\end{gathered}
$$

The fulfilment of these four requirements in a water SAD was evaluated in [53], identifying several limitations of optoelectronic control. A Teflon ring, which was coaxially positioned with the electrodes and holding eight radial fixed and uniformly distributed optical fibers, was placed around the arc zone (Figure 6(b)). The eight fibers were coupled to a single wide-area photodiode (P1). An additional optical fiber coupled to a second photodiode (P2), but similar to $\mathrm{P} 1$, was positioned in the bottom part of the ring between two of the previously mentioned fibers. The $\alpha, \Gamma$, and $T$ correlations were determined using the described setup.

3.6.2. Acoustic Control. The arc sound is produced by the intense vaporization of the anode material in speedily moving anode spots, where the current density is elevated [54]. The arc sound has been studied as a signal for the online estimation and monitoring of gas-metal arc welding [55-57]. Specialized welders have come into a consensus that the arc sound is essential to their capability to control the process [58]. Note that the current in gas-metal arc welding is an order superior to a typical current arc discharge in liquid for CNS synthesis. A method based on the sound level was suggested to gauge the arc voltage when the standard procedure (i.e., leads connected to the arc electrodes) was not viable [59]. This led to the question of whether or not sound could be used as a parameter to effectively achieve a closed-loop control in SAD. However, to the best of our knowledge, any intent of this type has not yet been reported for SAD.

3.6.3. Stabilization Using a Ballast Resistor. In the case of using electrical parameters like arc current or voltage to control the arc discharge, their voltage-current characteristic (U-I characteristic) should be thoroughly considered. To guarantee a stable arc, the total resistance of the circuit must be positive. Therefore, the external resistance should be bigger than the absolute value of the negative resistance of the discharge [60].

The arc discharge U-I characteristic using graphite electrodes in air is described as follows:

$$
\begin{gathered}
U=a+b s+\frac{c+s d}{I} \text { for } I<I \text { cri }(\text { silent } \operatorname{arc}), \\
U \approx \text { const.for } I>I \text { cri (hissing arc) }
\end{gathered}
$$

where $a, b, c$, and $k$ are constants; $s$ is the gap between the electrodes; and $d$ is the anode diameter.

The current in the silent arc interval seems to diverge when $I<I_{\text {cri }}$ (4); however, it is limited by the load line (Figure 7). The arc voltage drops to a lower level at a certain 


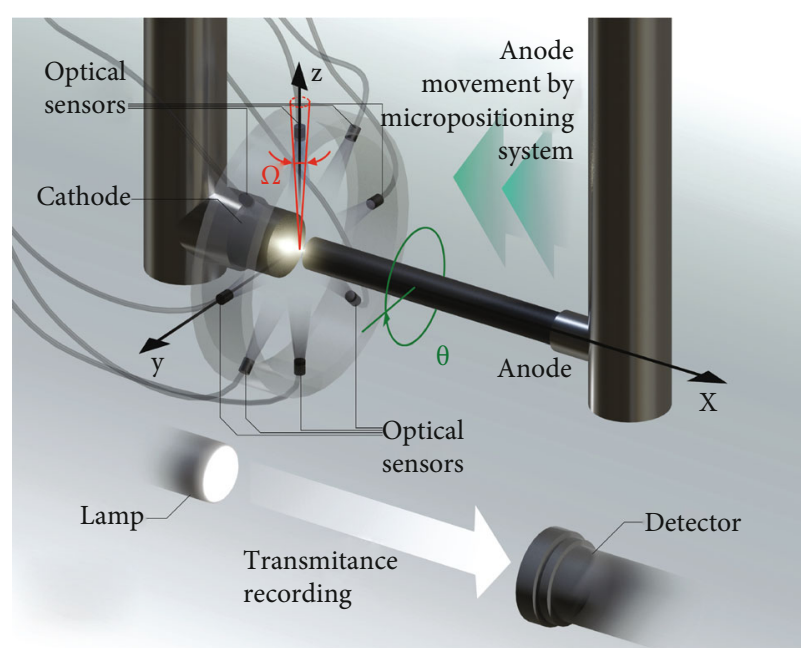

(a)

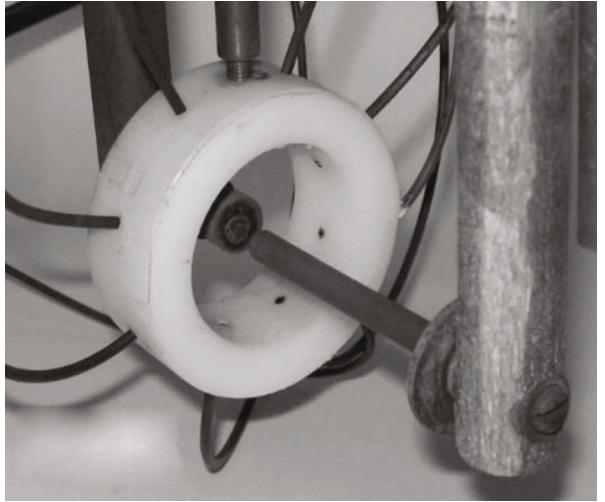

(b)

FIguRE 6: (a) Geometry of the experiment for the measurement of the $\alpha, \Gamma$, and $T$ parameters. The transmittance recording is performed using the light source $S$ and the detector $D$. (b) Device used to study the optical emission in SAD.

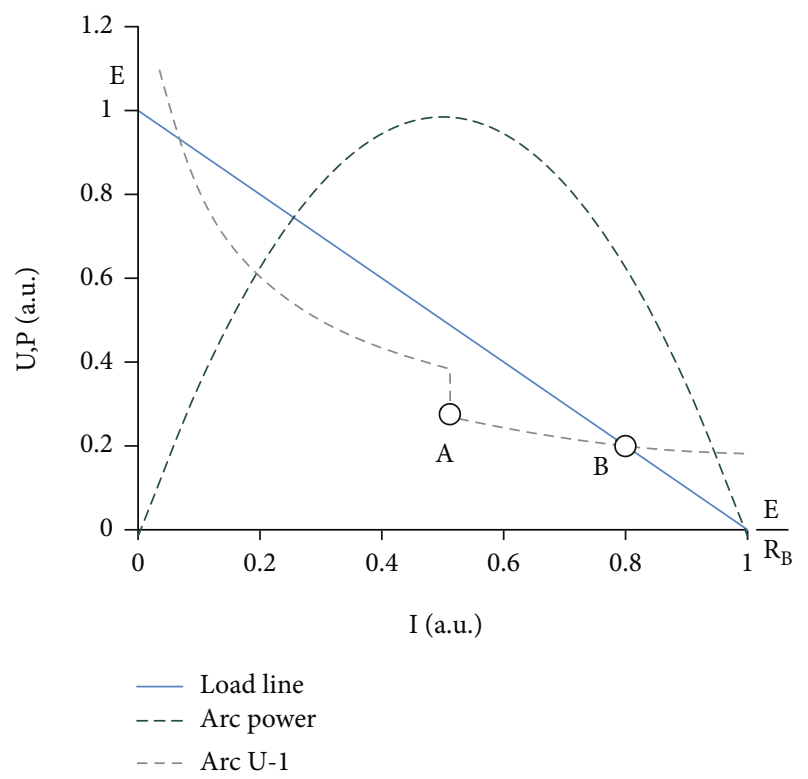

FIGURE 7: Electric arc voltage-current characteristic, circuit load line, and arc power curve.

critical arc current $\left(I_{\text {cri }}\right)$. The U-I curve becomes virtually horizontal, and a typical hissing noise is perceived. In Figure $7, I_{\text {cri }}$ corresponds to the current in point $A$.

To the best of our knowledge, the U-I characteristic in a liquid media has not yet been reported. However, it is expected that in SAD, for $I>I_{\text {cri }}$, the U-I characteristic can also be described by equation (3). Such an analogy is logical because in both media, the charge carriers transport through a highly ionized gas channel. The power supply voltage $(E)$ and the maximum circuit current $\left(E / R_{b}\right)$ define the load line through which the arc U-I characteristic will move. According to [60], the point where the U-I curve intercepts the load line is the point of arc stability $B$ (Figure 7 ). Thus, the inclu- sion of a ballast resistor in the circuit helps obtain a stable arc and calculate and set a desirable U-I pair or arc power. This is a very common technique used in arc discharge in gases, which can also be applied to SAD.

As shown in Figure 7, the internal arc resistance $R_{i}=$ $(\mathrm{dU} / \mathrm{dI})_{i}$ is negative in any point $i$ of the arc U-I curve. The discharge is stable only if the total circuit resistance is positive ( $R_{\mathrm{LL}}+\mathrm{Ri}>0$, where $R_{\mathrm{LL}}$ is the load line tangent). Therefore, a suitable resistance $R$ placed in series with the electrodes is a simple technique of guaranteeing the arc stability $\left(R_{\mathrm{LL}}+R_{i}+R>0\right)$. Ref. [45] reported that the use of a ballast resistor $(0.7 \Omega / 4.2 \mathrm{~kW})$ improves the current and the power stability by 4 and 2.7 times, respectively.

3.6.4. Voltage Control. In some works, the arc voltage has been used as a feedback parameter for the SAD stability control $[6,61]$. In [6], an arc voltage difference of $\pm 5 \mathrm{~V}$ was kept by utilizing the appropriate resistance, such that beyond it, the motor moves in the opposite direction to preserve a narrow arc gap during the synthesis. In the CNT synthesis process in [61], the forward advance of the anode electrode was controlled by voltage with $0.1 \mathrm{~V}$ accuracy.

3.6.5. Current Control. The arc current is the most considered electrical parameter in works on $\mathrm{SAD}$, and many papers have used its value to classify the nanoparticle synthesis. The arc current has also been used as a feedback signal in SAD system stabilization [44, 62-65]. The U-I characteristic is almost parallel to the arc current axis; thus, it is easier to quantify the arc current than the arc voltage. In some works, the power supplies can set up the desired arc current. In others, the arc current was maintained by looking at a measuring instrument and correspondingly acting over the electrode gap. However, automatic systems must sense the arc current through a transducer, and the simplest of them all is a shunt resistor. It is a direct, cost-effective, and simple solution extensively used in power electronics and with reasonable accuracy. Shunt resistors are usually placed either 

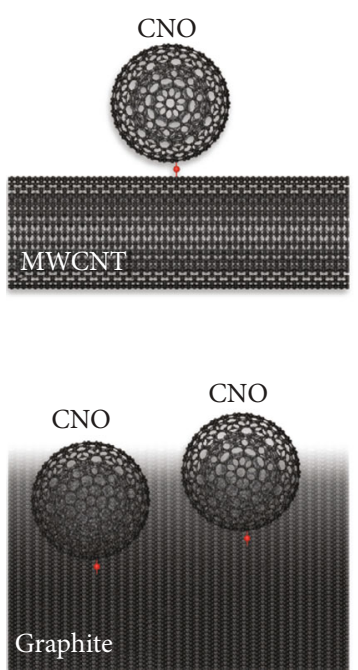
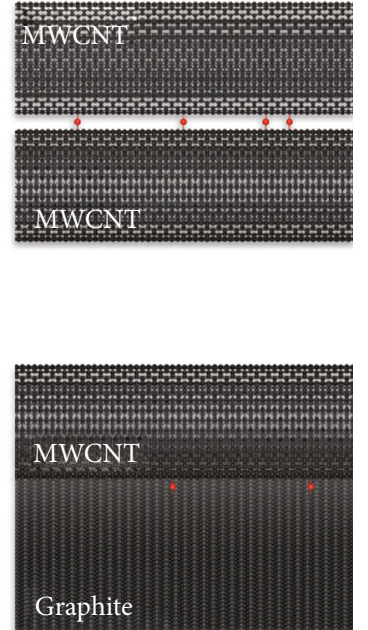

(a)

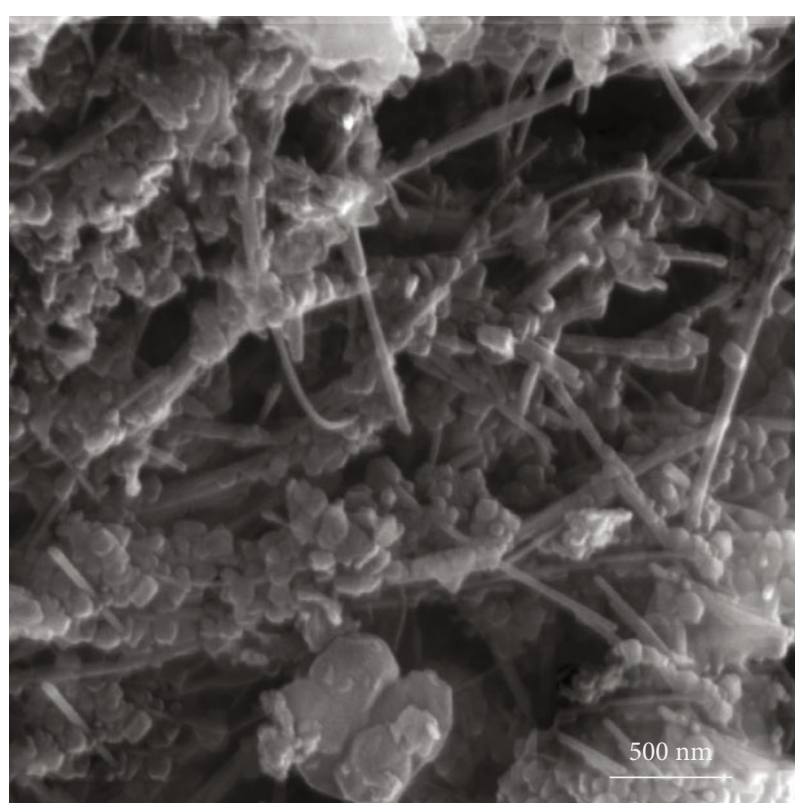

(b)

FIgURE 8: (a) Some of the possible aggregates formed in the precipitated products by ether bridges. (b) SEM image of the precipitated products, in which different nanoparticle cluster types are observed.

between the supply voltage and the load (high side) or between the load and the ground (low side). The voltage drop across the shunt is directly proportional to the arc current flow.

\section{Merits and Disadvantages of the Stabilization Methods}

The arc stability is harder to control in liquid than in gas. Section 2 describes the main sources of instability in SAD, while Section 3 presents the main stabilization methods described in the literature. Henceforward, the advantages and the disadvantages of the stabilization methods of the arc discharge will be discussed.

Using electrolytes to stabilize the SAD increases oxygen production via electrolysis, consequently intensifying the carbon loss in $\mathrm{CO}$ emission and forming the chemical bonds between nanostructures [62]. This could be the reason why in [22], the precipitate fraction increased. The $\mathrm{CNO}$, MWCNT, and graphite particles may be welded together by ether bridges (Figure 8). Furthermore, when obtaining pure CNSs, the purification process is more difficult because the salt and the additionally synthesized decorated nanoparticles must be eliminated. Although the arc was described as stable, no numerical data or comparison among syntheses on distilled water and electrolytes have been provided to support this statement. In contrast, part of the energy needed for the electric arc goes to the electrolytic process.

The authors that used the PREP method claimed that it leads to a better discharge stability; however, none of them have reported the current or power dispersion values. In [4], the synthesized material must be recovered from the cathode surface; therefore, a continuous production cannot be implemented. Neither the erosion rate of the electrodes nor the rigorous data on the nanostructure yield have been reported in these works. This method produces a large thermal stress in the anode tip caused by the large, fast, and periodic variation of the temperature. The thermal stress detaches graphite microparticles from the anode and is more prominent for the electrodes of big grains. In these works, the anode erosion rate was very high. Still assuming that the whole energy was wasted away in graphite evaporation, the evaporated mass represented a small fraction of the eroded anode mass. The yield values when the purification of the obtained material was completed were not reported. Therefore, the large anode erosion was probably caused by the crumbled graphite microparticles.

Given the intrinsic heterogeneity of the electrode material and the power oscillations, moving at a constant speed, the electrodes lead neither to their uniform erosion rate nor to a constant gap between them. Applying the method, the arc current gradually increases or decreases, leading in both cases to the discharge extinction (turned off or being short circuit). In this way, the discharges are neither achieved for prolonged periods nor stabilized [44].

In experiments without a control system or with fixed electrodes, the arc ignition procedure must be repeated several times if long synthesis times are desired. This increases the electrode erosion and the contaminants created at the moment the arc starts. Reaching a maximum of $38 \%$ in the power oscillations within the $\tau$ interval, the large dispersion $(\sim 30 \%)$ in both synthesis time and power values indicates that without stabilization, there is no reproducibility in the power values and topology of the electric and temperature fields. This variation of the synthesis conditions leads to a great diversity of the shapes and dimensions of the obtained nanostructures. The $\tau$ value depends on the electrical parameters of the system and the electrodes used. 


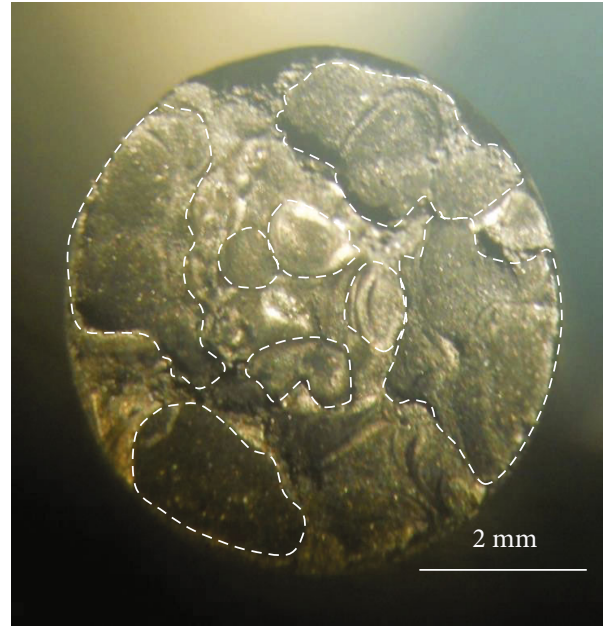

(a)

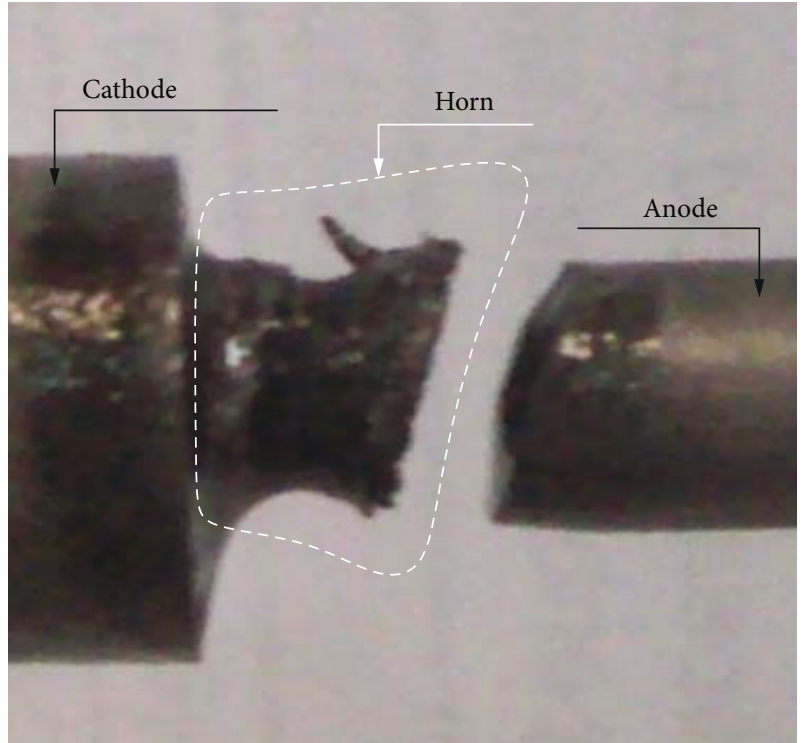

(b)

FIgure 9: (a) Anode tip surface after synthesis at $30 \mathrm{~A}$. The highlighted areas show the wear zones produced by the errant incidence of the electric arc. (b) Trumpet-shaped deposition at the front of the cathode produced at high current.

Manual control is not feasible because the variation time of the arc power is usually smaller than the reaction time (RT) of people. RT is the time interval between the presentation of a stimulus and the initiation of a muscular response to that stimulus. In humans, the average RT to a visual stimulus is $0.25 \mathrm{~s}$. Numerous factors affect the RT, which can comprise those related to factors dependent on the subject and those related to the stimulus in addition to those corresponding to the afferent system of the perception processing and response system. Among the first ones are physical state, warming, fatigue, and motivation. The other characteristics include age, gender, and substances administered (e.g., caffeine or drugs) [66]. Therefore, synthesis products with this type of "control" have a large dispersion in their morphology and dimensions.

The anisotropy of the emitted arc light, which is caused by the small gap between the electrodes, irregular erosion or depositions on the latter, light refraction and scattering in the bubbles, and strong liquid convection, makes it difficult to measure the light emission from a single observation point. Consequently, the optical emission in SAD does not correlate with the arc current.

Ref. [53] revealed that $\alpha$ does not obey a flat behavior because it may be awaited for isotropic emission. In the $30-50$ A current range, the electrode gap is small ( $\sim 0.7-$ $1 \mathrm{~mm}$ ), and the anode spot rapidly moves all over the anode front end surface as it wears out (Figure 9(a)). The anode wear is irregular, and the electrode gap is very narrow; hence, the arc light is not always visible neither with the same intensity from a single observation point. This is due to the refraction and scattering of light in the bubbles and the strong fluid convection. For cryogenic liquids, this constitutes a much more serious limitation than when using water because the bubbling is more intense. Meanwhile, for arc current densities bigger than $106 \mathrm{~A} \cdot \mathrm{cm}^{-2}$, a trumpet- shaped deposition on the cathode front surface modifies the gap geometry (Figure 9(b)). The number of dispersed CNSs in the liquid gradually increases during the synthesis; thus, the transmittance of the liquid media progressively decreases, adding one more difficulty to the optical regulation of the arc. This disadvantage can be overcome by continuously filtering the liquid $[52,67]$. However, in the case of the CNS synthesis, numerous products are formed. The filtration leads to product mixing, which results in a loss of the benefit of their natural separation, as would be the case of the separation of the floating hydrophobic products from those who precipitate. The optical radiation emitted by the arc undergoes intense absorption by the surrounding liquid media, and its emission spectrum displays a continuous shape due to the elevated temperature at the electrode gap. Unfortunately, a certain dedicated spectrum line that could be useful for the optical control was not found.

Several physical-chemical sound-generating processes occur during the SAD synthesis. Although acoustic emission is a valuable information source on the SAD processes that can be used to optimize the design of facilities, the application of the acoustic control method entails great difficulties because it is highly complex. So far, no acoustic method has been reported to control the SAD stability.

In suspensions, the sound is attenuated by the intrinsic absorption in the materials comprising the system and the viscous and thermal transport processes occurring at the interface of the inhomogeneities [68]. On the contrary, the presence of bubbles in a liquid suspension causes an increase in the effective bulk viscosity of the composite material directly proportional to the shear viscosity of the condensed phase. When using water and graphite electrodes, a suspension of the carbon particles of a wide range of sizes appears during the synthesis, and their concentration gradually 


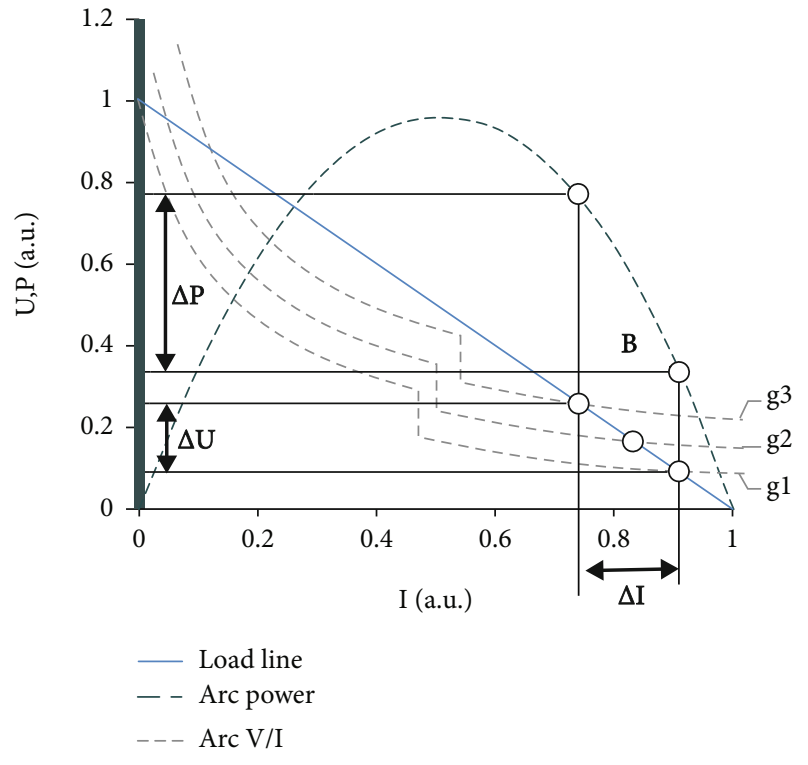

Figure 10: Power and voltage dependences on the arc current at different electrode gaps $(g 1<g 2<g 3)$.

increases. Thus, the sound absorption varies with time and affects the measuring accuracy. An intense bubbling containing a mixture of gases like $\mathrm{H}_{2} \mathrm{O}$ vapor, which is predominant, $\mathrm{CO}$, and $\mathrm{H}_{2}$ is created. A gas bubble is well known to disperse and absorb sound. These effects are very close to the resonant frequency of the bubble, which is the frequency at which the gas stiffness in the bubble is tuned by the mass reactance of the liquid around the bubble. Therefore, the liquid containing the gas bubbles is a dispersive medium [69]. Once SAD is started, a large part of the processes that generate sound emission occurs in a gaseous environment. The sound speed and the density in gases are lower than those in liquids, which induces a very strong acoustic impedance contrast at a gas-liquid interface. The sound transmission is usually very weak across a boundary with a strong impedance difference. Bearing in mind the acoustic field caused by a source in liquid or gas, the gas-liquid interface is commonly replaced by a perfectly reflecting boundary $[70,71]$. Ref. [29] reported on the relation between arc sound and other physical-chemical processes in SAD. The authors simultaneously recorded the arc current, voltage, and sound plus the anode displacement in a correlated manner. Several sound sources, such as background noise, bubbling, chemical reactions, mechanical vibrations, and carbon vapor jets, were identified. SAD was performed as an alternation of stable and unstable intervals. Likewise, in the case of optical control, no specific sound frequency was identified useful in controlling the arc discharge.

In the galvanic feedback control, any resistor present in the circuit will influence the load line slope and the point of arc stability. This is the case in electrode resistance, whose value not only is sometimes the same order of the ballast resistor but also varies during the synthesis. As the electrode is being eroded, its length decreases along with its resistance. Consequently, the load line slope changes. In contrast, a power supply has an output impedance that is usually not taken into account. SAD is a short circuit; thus, this resistance has a strong influence in the final electrical setup. Even when a ballast resistor is not ex professo included in the circuit, the sum of the power supply's output resistance and the electrode resistance acts instead of it. Finally, a shunt resistor is sometimes included in the circuit to gauge the arc current. Although its value is usually very small, the sum of all of the resistors present in the circuit should be considered as the total ballast resistor [44].

The studies, in which the voltage was used to control the discharge, did not provide information on the power supply used. They also did not provide details on the developed feedback mechanism. The electrode gap should be kept constant, and the arc U-I characteristic is almost perpendicular to the voltage axis; hence, very small changes in the arc voltage $(\Delta U)$ lead to big changes in the corresponding arc current or power, and a higher accuracy in quantifying the arc voltage in a very electrically noisy environment is demanded. With regard to the small changes in the arc voltage, the big changes in the corresponding arc current and power spread the particle size dispersion. Furthermore, bigger changes in the arc voltage will lead to a considerable variation in the arc gap.

Ref. [6] showed the great influence of the arc power on the structural quality and size of the produced CNSs. The author concluded that the increase in power is detrimental to the CNS production. Amorphous carbon and graphite fragments detached from the electrodes were observed only at high powers $(\sim 2 \mathrm{~kW})$. This result can be explained if we consider that the stability control by the voltage is ineffective $(\mathrm{dU} / \mathrm{dI} \approx 0)$. When the power grows, the vibrations and the thermal stress also increase. Consequently, the generation of such pollutants grows.

In using the arc voltage as a feedback signal, a higher accuracy in quantifying the arc voltage in a very electrically noisy environment is demanded because the arc U-I characteristic is almost perpendicular to the voltage axis, and, consequently, very small changes in the arc voltage $(\Delta U)$ lead to big changes in the corresponding arc current or power. This requirement makes the signal conditioning and front-end electronics more complex. With regard to the small changes in the arc voltage, the big changes in the corresponding arc current and power spread the particle size dispersion. Furthermore, bigger changes in the arc voltage will lead to a considerable variation in the arc gap.

To be used as a control parameter, the current should be gauged appropriately. The use of shunt is a low-cost and simple solution with a reasonable accuracy. The U-I curve is almost parallel to the arc current axis; hence, the best affordable electrical parameter to be quantified and used for discharge control is the arc current instead of the arc voltage. However, the relation among the arc current, arc power, electrode gap, and ballast resistor should always be considered to obtain the desired electrical conditions from the experiments.

As explained before, the point where the U-I characteristic intercepts the load line is the point of arc stability. In other words, for a given load line and a given gap between the electrodes, the arc current, at which the electric arc would be stable, is only one. However, it is very common to see articles reporting synthesis at different arc currents 
claiming that they have maintained the same gap. This is not possible to achieve without changing the slope of the load line. A different gap is used for every synthesis arc current. Therefore, the resultant product is not only obtained at different electrical conditions but also at geometrical ones (i.e., the temperature and electric potential fields are different). Keeping the same load line, the arc current will be inversely proportional to the gap. Control systems usually use this dependence to achieve a stable arc current. That is, the arc current is controlled by varying the gap between the electrodes, which is also affected by anode erosion or cathode deposition. In the case of performing a synthesis at different arc currents, the point of arc stability $B$ is shifted to a new position alongside the load line (Figure 10). As shown in the figure, the bigger the arc current, the lower the arc power. This is why after the electrodes are stopped, a raise in the arc intensity is observed just before the arc turns off. This behavior is also observed when a control method is not used.

The current feedback is used to act over a micropositioning mechanism to regulate the gap (DC-servo or step motors). However, these mechanisms are not fast enough to quickly adjust the arc gap between the submerged electrodes to compensate for the arc current fluctuations. Therefore, the micropositioning mechanism must be complemented with an electrical stabilization element. Inserting a ballast resistor in the circuit helps obtain a stable arc and set the desirable U-I pair or arc power. However, the electrode resistance and the power supply output resistance should be considered. Not considering these elements could lead to trying to stabilize the arc at a current where it is not appropriated and having a different arc power for the same arc current. An electric stabilization element like a ballast resistor significantly improves the current and the power stability [45].

\section{Conclusions}

The arc power stability is a crucial factor to guarantee the CNS homogeneity and quality. The election of an appropriate stabilization method is an essential condition, but this is not enough. The set-up mechanical design and suitable electrodes should also be optimized.

Most of the published works did not employ stabilization methods. In a large part of those that used stabilization systems, data that quantify or demonstrate the SAD stability have not been reported. This circumstance explains the differences in the electrode erosion rate values, yield, and purity of the obtained nanostructures, and sorts of contaminants present in the synthesis.

Methods based on manual control and the use of electrolytes, constant speed, and electrode rotation are not effective and only generate a large amount of contaminants.

Optic and acoustic control methods are subject to serious limitations because they involve propagation in a turbulent media that undergo rapid variations in its properties. Radiations must cross the boundaries of environments with different properties and experience various absorption mechanisms. Furthermore, the liquid medium absorbs part of the emitted radiation spectrum and changes its transmittance during synthesis. Nevertheless, they can be used as a tool to investigate some arc discharge properties.

In the hissing arc zone, in which the nanoparticles are synthesized, the voltage remains approximately constant, regardless of the current values. Hence, the arc control based on voltage regulation is ineffective.

The SAD current control is the most suitable method for ensuring the arc power stability. It is a simple and useful method that should be complemented with a ballast resistor because the micropositioning mechanisms are not quick enough to adjust the arc gap to compensate for the arc power fluctuations.

\section{Data Availability}

No data.

\section{Conflicts of Interest}

The authors declare that there is no conflict of interest regarding the publication of this paper.

\section{Acknowledgments}

The research was supported by AENTA-Cuba (PNUOLU-71, 2014) and the Program for Basic Research (N@NO-C, 2015), MES-Cuba.

\section{References}

[1] K. D. Sattler, "Carbon Nanomaterials Sourcebook," in Nanoparticles, Nanocapsules, Nanofibers, Nanoporous Structures, and Nanocomposites, vol. II, CRC Press, Taylor \& Francis Group, Boca Raton London New York, 2016.

[2] V. Georgakilas, J. A. Perman, J. Tucek, and R. Zboril, "Broad family of carbon nanoallotropes: classification, chemistry, and applications of fullerenes, carbon dots, nanotubes, graphene, nanodiamonds, and combined superstructures," Chemical Reviews, vol. 115, no. 11, pp. 4744-4822, 2015.

[3] R. B. Mathur, B. P. Singh, and S. Pande, Carbon Nanomaterials-Synthesis, Structure, Properties and Applications, CRC Press, Taylor \& Francis Group, Boca Raton, 2017.

[4] F. Alessandro, A. Scarcello, M. B. Valverde et al., "Selective synthesis of turbostratic polyhedral carbon nano-onions by arc discharge in water," Nanotechnology, vol. 29, no. 32, p. 325601, 2018.

[5] S. Kim, Y. Song, T. Takahashi, T. Oh, and M. J. Heller, “An aqueous single reactor arc discharge process for the synthesis of graphene nanospheres," Small, vol. 11, no. 38, pp. 50415046, 2015.

[6] R. Borgohain, J. Yang, J. P. Selegue, and D. Y. Kim, “Controlled synthesis, efficient purification, and electrochemical characterization of arc-discharge carbon nano-onions," Carbon, vol. 66, pp. 272-284, 2014.

[7] S. Kim, Y. Song, J. Wright, and M. J. Heller, "Graphene bi- and trilayers produced by a novel aqueous arc discharge process," Carbon, vol. 102, pp. 339-345, 2016.

[8] S. Scalese, V. Scuderi, S. Bagiante et al., "Morphology and distribution of carbon nanostructures in a deposit produced by 
arc discharge in liquid nitrogen," Phys. E Low-dimensional Syst. Nanostructures, vol. 44, no. 6, pp. 1005-1008, 2012.

[9] K. Vasu, K. Pramoda, K. Moses, A. Govindaraj, and C. N. R. Rao, "Single-walled nanohorns and other nanocarbons generated by submerged arc discharge between carbon electrodes in liquid argon and other media," Materials Research Express, vol. 1, 2014.

[10] F. J. Chao-Mujica, L. Garcia-Hernández, S. Camacho-López et al., "Carbon quantum dots by submerged arc discharge in water: synthesis, characterization, and mechanism of formation," Journal of Applied Physics, vol. 129, no. 16, p. 163301, 2021.

[11] H. Lange, M. Sioda, A. Huczko, Y. Q. Zhu, H. W. Kroto, and D. R. M. Walton, "Nanocarbon production by arc discharge in water," Carbon, vol. 41, no. 8, pp. 1617-1623, 2003.

[12] N. Sano, H. Wang, I. Alexandrou et al., "Properties of carbon onions produced by an arc discharge in water," Journal of Applied Physics, vol. 92, no. 5, pp. 2783-2788, 2002.

[13] N. Sano, M. Naito, M. Chhowalla et al., "Pressure effects on nanotubes formation using the submerged arc in water method," Chemical Physics Letters, vol. 378, no. 1-2, pp. 2934, 2003.

[14] N. Sano, T. Charinpanitkul, T. Kanki, and W. Tanthapanichakoon, "Controlled synthesis of carbon nanoparticles by arc in water method with forced convective jet," Journal of Applied Physics, vol. 96, no. 1, pp. 645-649, 2004.

[15] N. Sano, T. Kikuchi, H. Wang, M. Chhowalla, and G. A. Amaratunga, "Carbon nanohorns hybridized with a metal-included nanocapsule," Carbon, vol. 42, no. 1, pp. 95-99, 2004.

[16] H. W. Zhu, X. S. Li, B. Jiang et al., "Formation of carbon nanotubes in water by the electric-arc technique," Chemical Physics Letters, vol. 366, no. 5-6, pp. 664-669, 2002.

[17] T. Okada, T. Kaneko, and R. Hatakeyama, "Conversion of toluene into carbon nanotubes using arc discharge plasmas in solution," Thin Solid Films, vol. 515, no. 9, pp. 4262-4265, 2007.

[18] S. Scalese, V. Scuderi, S. Bagiante et al., "Controlled synthesis of carbon nanotubes and linear $\mathrm{C}$ chains by arc discharge in liquid nitrogen," Journal of Applied Physics, vol. 107, no. 1, p. $014304,2010$.

[19] X. Li, H. Zhu, B. Jiang, J. Ding, C. Xu, and D. Wu, "High-yield synthesis of multi-walled carbon nanotubes by waterprotected arc discharge method," Carbon, vol. 41, no. 8, pp. 1664-1666, 2003.

[20] L. A. Montoro, R. C. Z. Lofrano, and J. M. Rosolen, "Synthesis of single-walled and multi-walled carbon nanotubes by arc-water method," Carbon, vol. 43, no. 1, pp. 200-203, 2005.

[21] T. Charinpanitkul, W. Tanthapanichakoon, and N. Sano, "Carbon nanostructures synthesized by arc discharge between carbon and iron electrodes in liquid nitrogen," Current Applied Physics, vol. 9, no. 3, pp. 629-632, 2009.

[22] S. D. Wang, M. H. Chang, K. M. Der Lan, C. C. Wu, J. J. Cheng, and H. K. Chang, "Synthesis of carbon nanotubes by arc discharge in sodium chloride solution," Carbon, vol. 43, no. 8 , pp. 1792-1795, 2005.

[23] T. Sugai, H. Omote, S. Bandow, N. Tanaka, and H. Shinohara, "Production of fullerenes and single-wall carbon nanotubes by high-temperature pulsed arc discharge," The Journal of Chemical Physics, vol. 112, no. 13, pp. 6000-6005, 2000.
[24] N. Arora and N. N. Sharma, "Arc discharge synthesis of carbon nanotubes: comprehensive review," Diamond and Related Materials, vol. 50, pp. 135-150, 2014.

[25] C. Corbella, S. Portal, M. N. Kundrapu, and M. Keidar, "Anodic arc discharge: why pulsed?," Physics of Plasmas, vol. 27, no. 5, p. 054501, 2020.

[26] Y. L. Hsin, K. C. Hwang, F. R. Chen, and J. J. Kai, "Production and in-situ metal filling of carbon nanotubes in water," Advanced Materials, vol. 13, no. 11, pp. 830-833, 2001.

[27] N. Koprinarov and M. Konstantinova, "Energy balance of DC arc discharge with closely situated electrodes," Romanian Reports in Physics, vol. 56, no. 9-10, pp. 1167-1172, 2011.

[28] H. O. Pierson, Handbook of Carbon, Graphite, Diamond and Fullerenes-Properties, Processing and Applications, Noyes Publications, New Jersey, 1993.

[29] L. Hernandez-Tabares, S. Fortune-Fabregas, F. J. Chao-Mujica et al., "Multiparametric diagnostic in the synthesis of carbon nanostructures via submerged arc discharge: stability, nucleation and yield," Journal of Applied Physics, vol. 126, no. 18, p. 183301, 2019.

[30] S. Yatom, R. S. Selinsky, B. E. Koel, and Y. Raitses, “'Synthesison' and 'synthesis-off modes of carbon arc operation during synthesis of carbon nanotubes," Carbon, vol. 125, pp. 336343, 2017.

[31] S. Yatom, J. Bak, A. Khrabryi, and Y. Raitses, "Detection of nanoparticles in carbon arc discharge with laser-induced incandescence," Carbon, vol. 117, pp. 154-162, 2017.

[32] D. Bera, S. C. Kuiry, M. McCutchen et al., "In-situ synthesis of palladium nanoparticles-filled carbon nanotubes using arcdischarge in solution," Chemical Physics Letters, vol. 386, no. 4-6, pp. 364-368, 2004.

[33] A. D. Kiadehi, M. Jahanshahi, M. R. Mozdianfard, G. H. R. Vakili-Nezhaad, and R. Jabari Seresht, "Influence of the solution temperature on carbon nanotube formation by arc discharge method," Journal of Experimental Nanoscience, vol. 6, no. 4, pp. 432-440, 2011.

[34] D. M. Gattia, M. Vittori Antisari, and R. Marazzi, "AC arc discharge synthesis of single-walled nanohorns and highly convoluted graphene sheets," Nanotechnology, vol. 18, no. 25, p. 255604, 2007.

[35] S. Jong Lee, H. Koo Baik, J. E. Yoo, and J. H. Han, "Large scale synthesis of carbon nanotubes by plasma rotating arc discharge technique," Diamond and Related Materials, vol. 11, no. 3-6, pp. 914-917, 2002.

[36] R. Joshi, J. Engstler, P. K. Nair, P. Haridoss, and J. J. Schneider, "High yield formation of carbon nanotubes using a rotating cathode in open air," Diamond and Related Materials, vol. 17, no. 6, pp. 913-919, 2008.

[37] Y. A. Kim, H. Muramatsu, T. Hayashi, and M. Endo, "Catalytic metal-free formation of multi-walled carbon nanotubes in atmospheric arc discharge," Carbon, vol. 50, no. 12, pp. 4588-4595, 2012.

[38] J. Zhao, L. Wei, Z. Yang, and Y. Zhang, "Continuous and low-cost synthesis of high-quality multi-walled carbon nanotubes by arc discharge in air," Physica E: Lowdimensional Systems and Nanostructures, vol. 44, no. 7-8, pp. 1639-1643, 2012.

[39] M. Gattia, M. V. Antisan, R. Marazzi, L. Pilloni, V. Contini, and A. Montone, "Arc-discharge synthesis of carbon nanohorns and multiwalled carbon nanotubes," Materials Science Forum, vol. 518, pp. 23-28, 2006. 
[40] D. Zhang, K. Ye, Y. Yao et al., "Controllable synthesis of carbon nanomaterials by direct current arc discharge from the inner wall of the chamber," Carbon, vol. 142, pp. 278-284, 2019.

[41] J. C. Bae, Y. J. Yoon, H. K. Baik, S. J. Lee, and K. M. Song, "Effect of a rotating electrode on the formation of singlewalled carbon nanotubes," Applied Physics Letters, vol. 82, no. 13, pp. 2154-2156, 2003.

[42] C. S. Kim, S. M. Jeong, W. H. Koo, H. K. Baik, S. J. Lee, and K. M. Song, "Synthesis of B-C-N nanotubes by means of gas arc discharge with a rotating anode," Materials Letters, vol. 58, no. 22-23, pp. 2878-2881, 2004.

[43] D. N. Borisenko, N. N. Kolesnikov, M. P. Kulakov, and V. V. Kveder, "Growth of carbon nanotubes (CNTs) in electric-arc discharge in argon," International Journal of Nanoscience, vol. 1, no. 3 \& 4, pp. 235-246, 2002.

[44] L. Hernández-Tabares, E. Carrillo-Barroso, J. G. Darias-González, L. F. Desdín-García, R. J. Castillo-Torres, and M. Ramos-Aruca, "Arc current control for a carbon nanoparticle synthesis station," Revista Cubana de Física, vol. 28, 2011.

[45] L. Hernández-Tabares, J. G. Darias-González, J. Arteche-Díaz, E. Carrillo-Barroso, L. M. Ledo-Pereda, and L. F. Desdín-García, "Automated system for the synthesis of nanostructures via arc-discharge in liquids," Advances in Natural Sciences: Nanoscience and Nanotechnology, vol. 9, no. 3, 2018.

[46] A. Ashraf, K. Yaqub, S. Javeed et al., "Sublimation of graphite in continuous and pulsed arc discharges," Turkish Journal of Physics, vol. 34, no. 1, pp. 33-42, 2010.

[47] H. Lange, K. Saidane, M. Razafinimanana, and A. Gleizes, "Temperatures and C2 column densities in a carbon arc plasma," Journal of Physics D: Applied Physics, vol. 32, no. 9, pp. 1024-1030, 1999.

[48] F. Liang, M. Tanaka, S. Choi, and T. Watanabe, Electrode Temperature Measurement for Carbon Nanomaterial Production by Arc Discharge Method, International Plasma Chemistry Society, 2013.

[49] H. Lange, P. Baranowski, A. Huczko, and P. Byszewski, “An optoelectronic control of arc gap during formation of fullerenes and carbon nanotubes," The Review of Scientific Instruments, vol. 68, no. 10, pp. 3723-3727, 1997.

[50] D. Delaportas, P. Svarnas, and I. Alexandrou, "Arc discharge in water for dielectric nanoparticle production," in 29th ICPIG, 2009.

[51] D. Delaportas, P. Svarnas, I. Alexandrou, A. Siokou, K. Black, and J. W. Bradley, " $\gamma$-Al2O3nanoparticle production by arcdischarge in water:in situdischarge characterization and nanoparticle investigation," Journal of Physics D: Applied Physics, vol. 42, no. 24, p. 245204, 2009.

[52] D. Bera, E. Brinley, S. C. Kuiry et al., "Optoelectronically automated system for carbon nanotubes synthesis via arcdischarge in solution," Review of scientific instruments, vol. 76, no. 3, p. 033903, 2005.

[53] J. G. Darias-González, L. Hernández-Tabares, E. Carrillo-Barroso, L. M. Ledo-Pereda, J. Arteche-Díaz, and L. F. DesdínGarcía, "Note: limitations of the optoelectronic control for carbon nanoparticles synthesis via arc-discharge in solution," Review of Scientific Instruments, vol. 85, no. 3, 2014.

[54] Y. P. Raizer, Gas Discharge Physics, Springer-Verlag, Berlin Heidelberg, 1991.

[55] H. Koga, Y. Ogawa, T. Morita, and T. Sumitomo, "Analysis of acoustic signals on $\mathrm{CO} 2$ arc welding," in The Fifth Interna- tional Offshore and Polar Engineering Conference, vol. 11 OnePetro.

[56] J. R. McCardle, The Analysis of Airborne Acoustics of SAW Using Neural Networks, Abington Publishing for TW, 1994.

[57] J. Prezelj and M. Čudina, "Noise as a signal for on-line estimation and monitoring of welding process," ACTA Acustica United with Acustica, vol. 89, no. 2, pp. 280-286, 2003.

[58] J. F. Lancaster, "The physics of fusion welding Part 1: the electric arc in welding," in IEE Proceedings, pp. 233-254, 1987.

[59] M. G. Drouet and F. Nadeau, "Acoustic measurement of the arc voltage applicable to arc welding and arc furnaces," Journal of Physics E, vol. 15, no. 3, pp. 268-269, 1982.

[60] V. I. Gaponov, Física Electrónica, Instituto del Libro, La Habana, 1968.

[61] M. Jahanshahi, J. Raoof, and R. Jabari Seresht, "Voltage effects on the production of nanocarbons by a unique arc-discharge set-up in solution," Journal of Experimental Nanoscience, vol. 4, no. 4, pp. 331-339, 2009.

[62] Z. E. Horváth, K. Kertész, L. Pethő et al., "Inexpensive, upscalable nanotube growth methods," Current Applied Physics, vol. 6, no. 2, pp. 135-140, 2006.

[63] H. Chang, T. T. Tsung, L. C. Chen et al., "TiO2 nanoparticle suspension preparation using ultrasonic vibration-assisted arc-submerged nanoparticle synthesis system (ASNSS)," Materials Transactions, vol. 45, no. 3, pp. 806-811, 2004.

[64] T. Charinpanitkul, K. Kanjanaprapakul, N. Leelaviwat, N. Kurukitkoson, and K. S. Kim, "Effect of arc current on characteristics of nanocarbons prepared by cryogenic arc discharge method," Journal of Industrial and Engineering Chemistry, vol. 16, no. 6, pp. 912-917, 2010.

[65] O. M. Awadallah, R. M. Rashad, and A. S. Wifi, "Study of the effect of anode/cathode geometry on the yield rate and quality of the MWCNTs synthesized by submerged arc discharging," in Volume 2: Systems; Micro and Nano Technologies; Sustainable Manufacturing, vol. 2, 2013.

[66] J. Pérez-Tejero, J. Soto-Rey, and J. J. Rojo-González, "Estudio del tiempo de reacción ante estímulos sonoros y visuales," European Journal of Human Movement, vol. 27, pp. 149-162, 2011.

[67] D. Bera, G. Johnston, H. Heinrich, and S. Seal, "A parametric study on the synthesis of carbon nanotubes through arcdischarge in water," Nanotechnology, vol. 17, no. 6, pp. 1722$1730,2006$.

[68] J. R. Allegra and S. A. Hawley, "Attenuation of sound in suspensions and emulsions: theory and experiments," The Journal of the Acoustical Society of America, vol. 51, no. 5B, pp. 15451564, 1972.

[69] D. T. Laird and P. M. Kendig, "Attenuation of sound in water containing air bubbles," The Journal of the Acoustical Society of America, vol. 23, no. 1, pp. 142-142, 1951.

[70] A. D. Pierce, Acoustics. An introduction to its physical principles and applications, AIP, New York, 1994.

[71] O. A. Godin, "Low-frequency sound transmission through a gas-liquid interface," The Journal of the Acoustical Society of America, vol. 123, no. 4, pp. 1866-1879, 2008. 